\title{
It is not only extending donor criteria: it is extending the donor pool. A cross- sectional survey from the European Society of Organ Transplantation
}

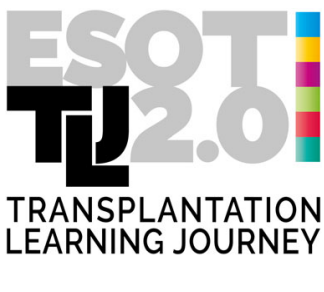

TRANSPLANTATION LEARNING JOURNEY

Nuria Masnou ${ }^{1}$ (D), Maria Irene Bellini ${ }^{2,3}$ Working Group Expanding the Donor Pool

Liset H. M. Pengel ${ }^{4}$ (D) Giuseppe Feltrin ${ }^{5}$ \& the TLJ 2.0

University Hospital Dr. Josep Trueta, Girona, Spain

2 Azienda Ospedaliera San Camillo Forlanini, Rome, Italy

3 Department of Surgical Sciences, Sapienza University, Rome, Italy 4 Nuffield Department of Surgical Sciences, Centre for Evidence in Transplantation, University of Oxford, Oxford, UK

5 Regional Centre for Transplant Coordination of the Veneto Region, Padua, Italy

E-mail: m.irene.bellini@gmail.com

Dear Editors,

As part of the TLJ2.0 organised by the European Society of Organ Transplantation (ESOT), our working group surveyed transplant professionals' attitudes towards expanding the donor pool, barriers and interventions to overcome these. The survey was posted for three weeks in July-August, 2020, via the ESOT's social media platforms.

A total of 135 participants, consisting mainly of surgeons $(34 \%)$, transplant coordinators $(30 \%)$, physicians (15\%) and intensivists (4\%) completed the survey (Table 1). Most respondents worked in the field for $>20$ years (34\%), 6-10 years (20\%) or $11-15$ years (19\%). The main European countries of practice were the Netherlands (16\%), the United Kingdom (10\%), Italy (8\%), Sweden (7\%) and Spain (4\%) (Q1-3).

History of cancer (Q4). The $88 \%$ felt that the history of leukaemia was not a contraindication to donate, although $15 \%$ suggested a multi-disciplinary team decision. Some stressed the need for explicit recipient consent $(4 \%)$ or clearly stating the risks to the recipient (3\%). Two respondents considered the history of leukaemia as a contraindication.

$H I V+$ donor for HIV+ recipient (Q5). The 77\% would accept grafts from HIV+ donors for HIV+ recipients. An evaluation of risks/benefits of this procedure and/or need for further tests was frequently mentioned.

Establishment of clinical protocols to support decisionmaking was deemed of great value, and respondents not willing to proceed stated it was too risky or referred to country-specific contraindications.

Table 1. Description of the survey questions

\begin{tabular}{ll}
\hline Survey questions & Responses $(n)$ \\
\hline Q1. Please indicate your country & 135 \\
Q2. What is your profession? & 135 \\
Q3. How many years of experience do you & 135 \\
have? & \\
Q4. You are on duty as a responsible for the & 97 \\
liver transplant unit and you receive a call & \\
offering a graft. Clinical history: 35 years & \\
old, traumatic brain injury, 4 ICU days. Past & \\
history of Leukaemia (as a teenager), & \\
15 years free of disease. Suppose there is a & \\
rare blood group and that you have a & \\
patient pending for transplantation for & \\
more than 2 years because of the blood & \\
group. Would you accept this graft? & \\
Q5. Some countries are grafting HIV+ & 86 \\
donors for HIV+ recipients. In order to & \\
expand the donor pool. Would you be in \\
favour of this option?
\end{tabular}


$\mathrm{HCV}+$ donor for $\mathrm{HCV}-$ recipient (Q6). The 59\% would accept grafts from $\mathrm{HCV}+$ donors for HCVrecipients, mainly due to the availability of effective anti-viral treatment. Respondents against accepting these grafts reported it was not a justifiable additional risk to patients, and stated ethical concerns and expensive treatment.

Continuation of transplantation programmes during the COVID-19 pandemic (Q7-8): During the COVID19 outbreak, $60 \%$ of the respondents' centres put their transplant activity on hold. This was done for a short time or certain donor categories, e.g. living or older donors, or recipients, e.g. patients with acute indications. The mean reason for closure was shortage of ICU beds, staff or testing capacities. The $78 \%$ stated that their hospital has defined a national or local continuation plan.

Intensive care to facilitate organ donation (ICOD) and its barriers (Q9). Only $38 \%$ had experience with ICOD.
Identified barriers were financial and organisational, lack of public knowledge regarding brain death, lack of competent staff, and ethical and legal country-specific barriers.

Donor management of a DBD donor (Q10). Respondents were equally divided between those who begin when switching from curative treatment to palliative care, and those who wait for determination of brain death.

In summary, many transplant professionals were willing to accept donors with an additional risk of transmission of infectious or malignant disease although there was no consensus on clinical pathways [1-3]. Many centres closed during the height of the pandemic and now have continuation plans in place [4-6]. Despite ICOD's potential to increase the donor pool $[7,8]$, the majority of respondents had no experience. The uncertainties highlighted by the survey will be further explored in an opinion paper that is currently in preparation by the working group.

\section{REFERENCES}

1. Eccher A, Girolami I, Marletta S, et al. Donor-transmitted cancers in transplanted livers: analysis of clinical outcomes. Liver Transpl 2021; 27: 55.

2. Muller E, Botha FCJ, Barday ZA, Manning K, Chin-Hong P, Stock P. Kidney Transplantation in HIV positive patients: current practice and management strategies. Transplantation 2020. https://doi.org/10. 1097/TP.0000000000003485 Epub ahead of print.

3. Sise ME, Goldberg DS, Kort JJ, et al. Multicenter study to transplant hepatitis C-infected kidneys (MYTHIC): an open-label study of combined glecapre- vir and pibrentasvir to treat recipients of transplanted kidneys from deceased donors with hepatitis $\mathrm{C}$ virus infection. J Am Soc Nephrol 2020; 31: 2678.

4. Bellini MI, Tortorici F, Capogni M. COVID-19 in solid organ transplantation: an analysis of the impact on transplant activity and wait lists. Transpl Int 2021; 34: 209.

5. Bellini MI, Tortorici F, Capogni M. Kidney transplantation and the lockdown effect. Transpl Int 2020; 33: 1142.

6. Bellini MI, Tortorici F, Capogni M. Resuming elective surgical activity after the COVID-19 wave: what the patients need to know. Br J Surg 2020; 107: e345e346. https://doi.org/10.1002/bjs.11802

7. Martín-Delgado MC, Martínez-Soba F, Masnou N, et al. Summary of Spanish recommendations on intensive care to facilitate organ donation. Am J Transplant 2019; 19: 1782.

8. Domínguez-Gil B, Coll E, Elizalde J, et al. Expanding the donor pool through intensive care to facilitate organ donation: results of a spanish multicenter study. Transplantation 2017; 101: e265. 